\title{
Inhibition of protein kinase C activity inhibits osteosarcoma metastasis
}

\author{
He-Jun $\mathrm{Hu}^{1}$, Xiong-Wei Deng ${ }^{1}$, Run-Xiang Li ${ }^{1}$, De-Wang Chen ${ }^{1}$, Chao Xue ${ }^{2}$
}

${ }^{1}$ Nanchang Hongdu Hospital of Traditional Chinese Medicine, Beijing, China
${ }^{2}$ Chinese General PLA Hospital, Beijing, China

Submitted: 17 April 2018

Accepted: 21 August 2018

Arch Med Sci 2019; 15 (4): 1028-1034

DOI: https://doi.org/10.5114/aoms.2018.79450

Copyright $\odot 2018$ Termedia \& Banach

\section{Abstract}

Introduction: For some cancers bone is the preferred site for metastasis and involves a cascade involving transition of epithelial cells to mesenchymal cells and subsequent intravasation to the blood and lymph vessels, and finally hematogenous dissemination to perivascular niches of the bone marrow sinusoids. It has been shown that protein kinase $C$ can aid metastasis to bone. Hence, pharmacological inhibition of protein kinase C (PKC) activity is thought of as a potential therapeutic option in bone metastatic lesions. The objective of the current study was to investigate how PKCs exert their effect on bone cancer metastasis and to test the efficacy of pharmacological inhibition of PKC on bone metastasis.

Material and methods: The effect of the PKC inhibitor G06983 on epithelial and mesenchymal cell marker expression in the osteosarcoma cell line DAN was determined by immunoblot and immunofluorescence analysis. The in vivo effect of G06983 was evaluated with a xenograft model using DAN cells. Results: Treatment with transforming growth factor $\beta$ (TGF- $\beta$ ) led to loss of the epithelial cell marker and gain of mesenchymal cell markers in the osteosarcoma cell line, DAN. This transition occurred concomitantly with PKC activation. TGF- $\beta$-mediated PKC activation resulted in activation of ribosomal protein 6 (S6), but not S6K1. Pharmacological inhibition of PKC activation attenuated these effects. In a xenograft model of experimental metastasis, pharmacological inhibition of PKC activation over a period of 4 weeks reduced both tumor burden and metastasis to lungs.

Conclusions: Our results indicate that PKC potentiates tumor metastasis to the bone by potentiating translation increase and can be putatively inhibited by pharmacological inhibition.

Key words: bone metastasis, osteosarcoma, protein kinase C, pharmacological inhibition.

\section{Introduction}

Bone is a preferred site for metastasis for numerous cancers, including breast and prostate [1]. Metastasis to bone involves initial transition of epithelial cells to mesenchymal cells and subsequent intravasation to the blood and lymph vessels, and finally hematogenous dissemination to perivascular niches of the bone marrow sinusoids, which are also the site of formation of the metastatic lesions [2]. Protein kinase C epsilon (PKC $\varepsilon$ ) has been shown to potentiate both metastasis to bone and the resultant pain in the bone via the transient receptor potential vanilloid 1 (TRPV1) receptor [2, 3].

\author{
Corresponding author: \\ Dr. Chao Xue \\ Chinese General \\ PLA Hospital \\ 28 Fuxing Road \\ Haidian District \\ 100853 Beijing, China \\ Phone: +86 1066887329 \\ E-mail: chaoxue_med12@ \\ 126.com
}


The PKC is a family of serine-threonine kinases that have been shown to regulate different aspects of differentiation, proliferation, motility, and apoptosis in cells. This family has ten structurally related isozymes that have been classified as classical or conventional PKCs $-\alpha, \beta \mid, \beta I$, and $\gamma$; novel PKCs $-\delta, \varepsilon, \eta$, and $\phi$; and atypical PKCs $-\zeta$, and $\lambda / \mathrm{l}$. Historically even though PKCs have been categorized as oncogenes, recent research has shown that individual isozyme has a context-dependent action as either a pro-oncogenic or an anti-carcinogenic effector [2, 4-6]. However, PKC $\varepsilon$ has been shown to function as a potential tumor biomarker and oncogenic kinase in all cases, inclusive of breast, lung, prostate, and head and neck cancer in both in vitro and in vivo models [7-14]. Pharmacological PKCe inhibition is thus being touted as a potential therapeutic option in a multitude of cancer metastases [14, 15].

However, the precise mechanisms by which PKCs exert their effect have not been completely defined yet. Hence, the objective of the current study was to investigate how PKCs exert their effect on bone cancer metastasis and to test the effect of pharmacological inhibition of PKC on bone metastasis. Our results reveal that PKC activation actually causes global translational upregulation whereas its inhibition almost completely attenuates bone metastasis in a xenograft model.

\section{Material and methods}

\section{Cell culture and treatment}

The DAN (CRL-2130) cell line was purchased from the ATCC (Manassas, VA, USA) and cultured in RPMI 1640 medium supplemented with $10 \%$ fetal bovine serum (FBS) (Thermo Fisher Scientific, Shanghai, China) and $100 \mathrm{lU} / \mathrm{ml}$ penicillin and 100 $\mu \mathrm{g} / \mathrm{ml}$ streptomycin (Thermo Fisher Scientific). The cells were treated with 10 nM Go6983 (Selleckchem, Texas, USA), $10 \mathrm{nM}$ bisindolylmaleimide IX (Bis IX) (Sigma-Aldrich), or the Akti-1/2 inhibitor (Abcam, Waltham, MA, USA) for indicated times to cell harvest and analysis.

\section{Cell lysis and immunoblot analysis}

To perform immunoblot analysis, Pierce RIPA lysis buffer (Thermo Fisher Scientific) was used to lyse the cells. The lysis buffer was supplemented with mini protease inhibitor cocktail (Roche Diagnostics, Indianapolis, IN, USA) and phosphatase inhibitor cocktails 2 and 3 (Sigma-Aldrich Shanghai Trading Co Ltd, Shanghai, China). Thirty micrograms of protein lysates were resolved by SDS-PAGE. Membranes were probed with indicated primary antibodies at $1: 1000$ dilution and detected by chemiluminescence. Anti-E-cadherin, N-cadherin, P-PKC (Y311), Total PKC, P-Akt
(S473), Total Akt, P-S6K1 (T389), Total S6K1, P-S6 (S240/244), and Total S6 antibodies were purchased from Cell Signaling Technology (USA). Anti-fibronectin and $\beta$-actin antibodies were purchased from Abcam (Waltham, MA, USA). Antipuromycin antibody was obtained from Kerafast (Boston, MA, USA).

\section{Immunofluorescence analysis}

DAN cells were grown on glass coverslips in 24well plates overnight prior to treatment with TGF- $\beta$ ( $5 \mathrm{ng} / \mathrm{ml}$; Roche Diagnostics) for $72 \mathrm{~h}$. Cells were treated with $1 \mathrm{nM}$ Go6983 or DMSO for the last $24 \mathrm{~h}$. Cells were then washed with PBS and fixed with $4 \%$ paraformaldehyde (in PBS) at room temperature (RT) for $15 \mathrm{~min}$ or with ice-cold methanol at room temperature (RT) for $7 \mathrm{~min}$. Cells were then washed three times with PBS prior to permeabilization with $0.1 \%$ Triton $\mathrm{X}-100$ in PBS for $5 \mathrm{~min}$. Permeabilized cells were washed five times with PBS and blocked with blocking reagent (0.1\% saponin, $10 \%$ goat serum in PBS) for $1 \mathrm{~h}$ at room temperature (RT). Cells were then washed once with PBS, followed by incubation with antiE-cadherin (1: 500) and anti-P-SMAD2/3 (1:200; Cell Signaling Technology) primary antibodies in blocking reagent overnight at $4^{\circ} \mathrm{C}$. Cells were washed three times with PBS and incubated with labeled secondary antibodies for $1 \mathrm{~h}$ at RT in the dark. Cells were then washed five times with PBS and coverslips were mounted with Vectashield containing DAPI ( $\mathrm{H}-1200)$ prior to microscopy. Images were acquired through a $20 \times$ objective with a Zeiss 710 confocal laser microscope (Zeiss, Oberkochen, Germany). Representative images are shown in all figures at the same exposure and magnification and in merged color images; co-localization is indicated by yellow and orange regions.

\section{Xenograft assay}

Experiments were approved by the Institutional Animal Care and Use Committee of the Chinese General PLA Hospital. For xenograft assays, $10^{6}$ firefly luciferase expressing DAN cells were injected via the tail vein of six-week-old female $p 53^{--}$ mice ( $n=5$ per group). One day after the initial injection, mice were injected with either DMSO or $10 \mathrm{nM} / \mathrm{kg}$ body weight of Go6983 (once every day). Mice were assessed weekly for tumor formation up to 28 days using in vivo bioluminescence imaging using an IVIS imaging system (IVIS Imaging System 200, Xenogen Corporation, PerkinElmer, Waltham, USA) fitted with an ultrasensitive CCD camera. After euthanasia of mice on day 28 , lungs were excised and imaged by bioluminescence imaging to detect metastatic lesions. 


\section{Ehics}

Experiments were approved by the Institutional Animal Care and Use Committee of Nanchang Hongdu Hospital of Traditional Chinese Medicine.

\section{Results}

The PKC activity has been shown to be centrally related to bone cancer metastasis $[2,3]$ and transforming growth factor $\beta$ (TGF- $\beta$ ) is known to activate PKC [16]. Moreover, a central requirement of metastasis is downregulation of the epithelial cell marker E-cadherin and gain of mesenchymal cell markers such as fibronectin and $\mathrm{N}$-cadherin [17]. Hence, to determine whether treatment with TGF- $\beta$ of the osteosarcoma cell line DAN would induce changes in epithelial and mesenchymal cell markers, DAN cells were treated with TGF- $\beta$ over

A

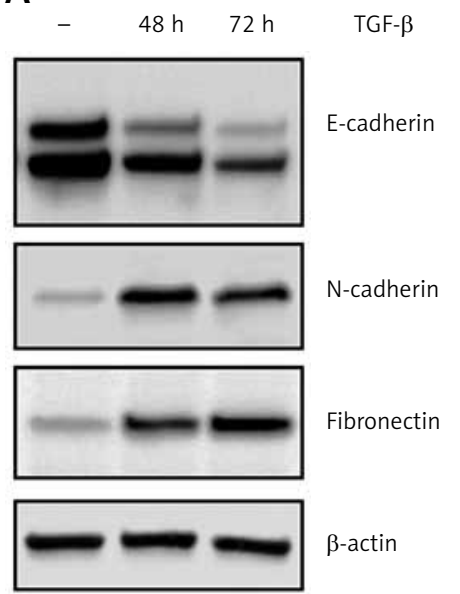

B

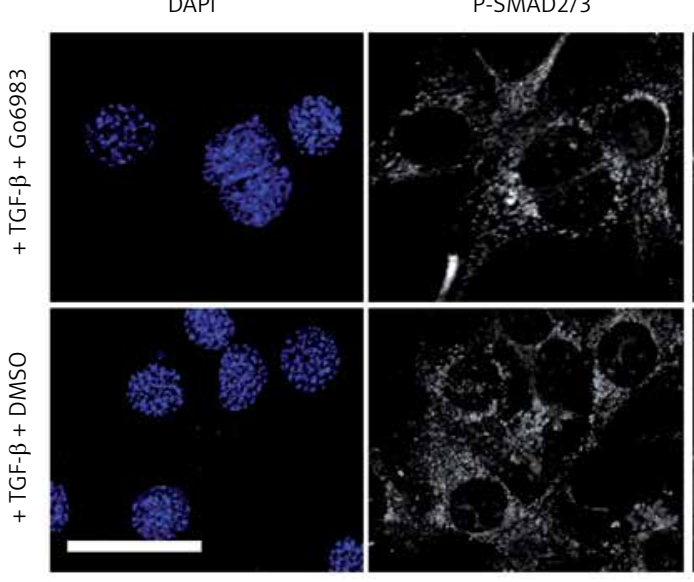

a period of $72 \mathrm{~h}$ and cell lysates were probed for $\mathrm{E}$-cadherin, fibronectin, and $\mathrm{N}$-cadherin. Whereas TGF- $\beta$ resulted in significant loss of E-cadherin expression, it also resulted in concomitant upregulation of fibronectin and $\mathrm{N}$-cadherin (Figure $1 \mathrm{~A}$ ), indicating that TGF- $\beta$ treatment does induce a mesenchymal phenotype in these cells. To next determine if this switch between epithelial and mesenchymal phenotype was being mediated by PKC activation, we treated cells with the PKC inhibitor G06983 [18] for the last 24 h of TGF- $\beta$ treatment and then performed immunofluorescence for E-cadherin. Treatment with G06983 prevented $\mathrm{E}$-cadherin expression from being downregulated, even though it did not affect TGF- $\beta$ activation as evident by P-Smad2/3 staining (Figures 1 B, C). Treatment with Go6983 also inhibited induction of mesenchymal cell markers, $\mathrm{N}$-cadher-
C

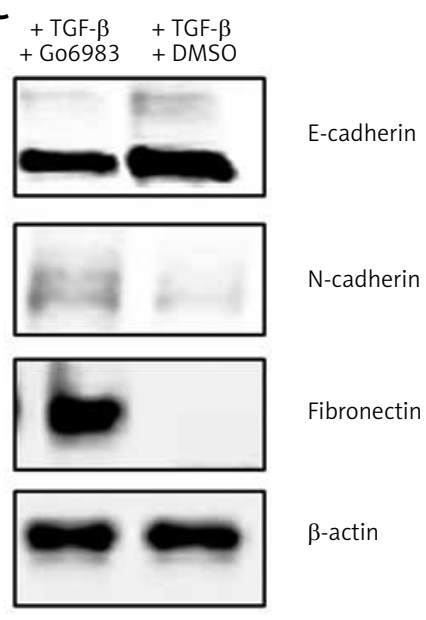

E-cadherin

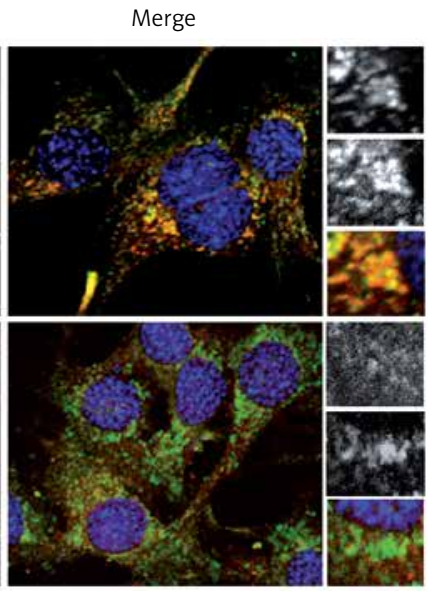

Figure 1. Treatment with TGF- $\beta$ leads to a switch from epithelial to mesenchymal cell marker in the osteosarcoma cell line, which can be inhibited by pharmacological inhibition of PKC. A - Immunoblot analysis of indicated markers in cell lysates obtained from DAN cells treated with TGF- $\beta$ for $72 \mathrm{~h}$. The blots were stripped and re-probed with anti- $\beta$-actin to confirm equal loading. Representative blots from three independent experiments are shown. B - Immunofluorescence analysis of E-cadherin and P-Smad2/3 in DAN cells treated with TGF- $\beta$ for $72 \mathrm{~h}$ and with $10 \mathrm{nM}$ of G06983 or DMSO in hours 48-72. Scale bar: $10 \mu \mathrm{m}$. Images from three independent experiments are shown. C - Immunoblot analysis of indicated markers in cell lysates obtained from DAN cells treated with TGF- $\beta$ for $72 \mathrm{~h}$ and with $10 \mathrm{nM}$ of Go6983 or DMSO from hours 48-72. The blots were stripped and re-probed with anti$\beta$-actin to confirm equal loading 
in and fibronectin (Figure $1 \mathrm{C}$ ). However, once cells underwent epithelial-mesenchymal transition (EMT), treatment with the PKC inhibitor G06983 could not revert the cells to an epithelial phenotype (data not shown). Of note, no significant cell death was observed within $24 \mathrm{~h}$ of treatment with the PKC inhibitor at the indicated dose.

To determine whether TGF- $\beta$ treatment causes PKC activation in DAN cells, we determined P-PKC (Y311) levels in DAN cells \pm TGF- $\beta$ treatment and saw robust induction of P-PKC (Figure $2 \mathrm{~A}$ ). This induction was prevented by prior treatment with either G06983 or another pan-PKC inhibitor, Bis IX (Figure $2 \mathrm{~A}$ ). Even the endogenous PKC activity seen in DAN cells (Figure 2 B) can be completely inhibited by prior treatment with Go6983 (Figure 2 C).

The TGF- $\beta$ treatment is known to induce Akt activation [19]; however, the effect of G06983 or Bis IX on PKC activation was independent of changes in Akt activation as assessed by P-Akt (S473) expression (Figure $2 \mathrm{~A}$ ), indicating that activation of PKC is either downstream of Akt activation or

A

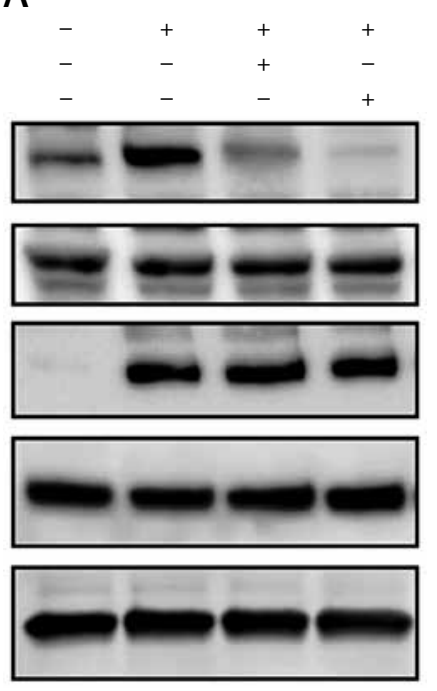

TGF- $\beta$ (72 h)

Go6983

Bis-IX

P-PKC (Y311)

Total PKC

P-Akt (S473)

T-Akt

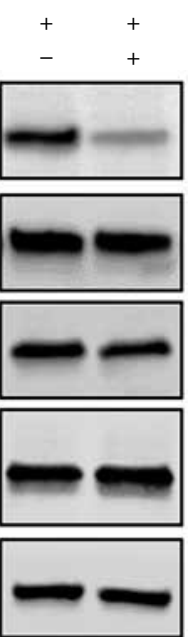

TGF- $\beta$ (72 h)

Akt-1/2

P-Akt (S473)

T-Akt

P-PKC (Y311)

Total PKC

$\beta$-actin

DAPI

B
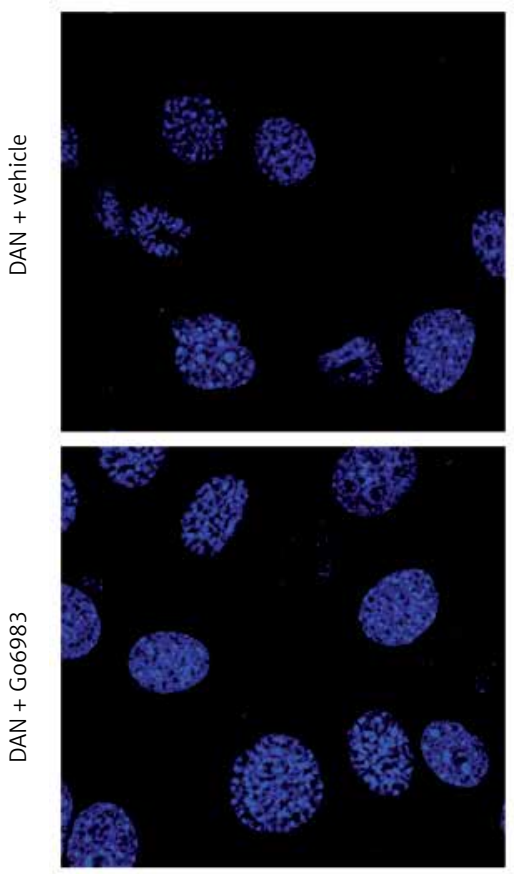

P-PKC (Y311)
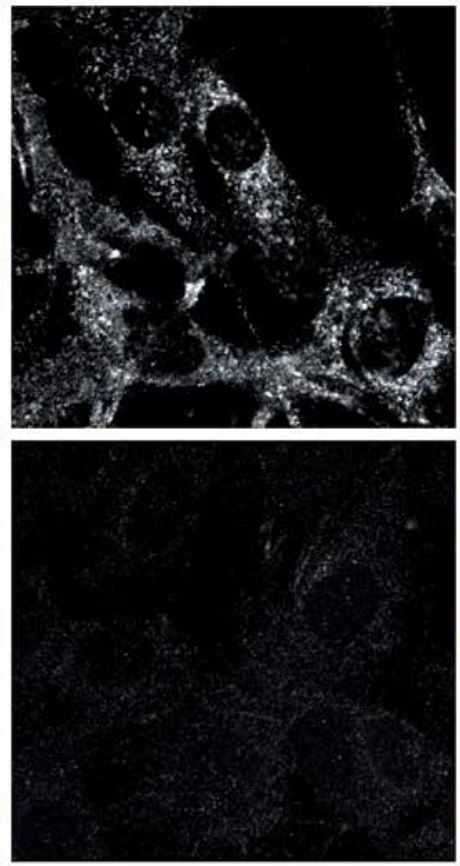
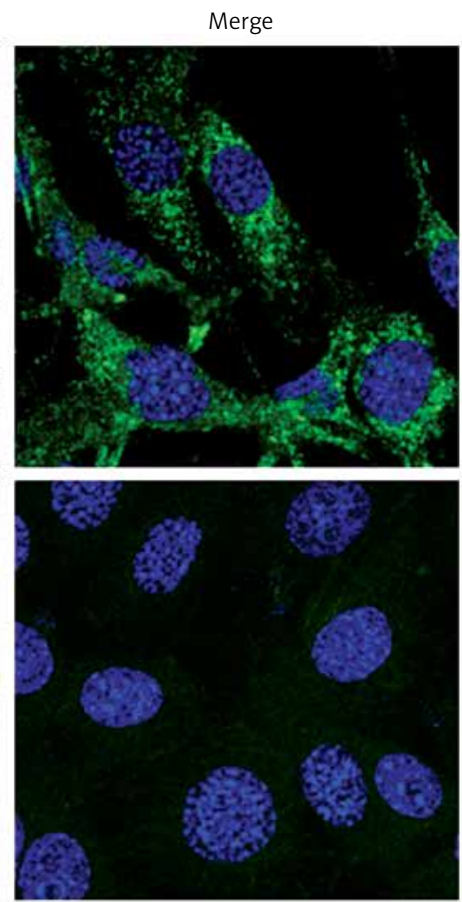

Figure 2. TGF- $\beta$ treatment leads to increased PKC-activation which can be effectively attenuated by pharmacological inhibition of PKC. A - Immunoblot analysis of indicated markers in PKC activation (P-PKC, Y311), and Akt activation (P-Akt, S473) in cell lysates obtained from DAN cells treated with TGF- $\beta$ for $72 \mathrm{~h} \pm$ PKC inhibitors Go6983 or Bis IX. The blots were stripped and re-probed with anti- $\beta$-actin to confirm equal loading. Representative blots from three independent experiments are shown. B - Immunofluorescence analysis of basal PKC activation in DAN cells $\pm 10 \mathrm{nM}$ of Go6983 for $30 \mathrm{~min}$. Scale bar: $10 \mu \mathrm{m}$. Images from three independent experiments are shown 
is independent of Akt activation. To determine whether Akt activation occurs upstream of PKC activation, DAN cells were treated with the Akt inhibitor Akti-1/2. Even though Akti-1/2 inhibited Akt activation and EMT, it did not have any effect on P-PKC (Figure $2 \mathrm{D}$ ), indicating that Akt and PKC activation occurs by independent pathways following treatment with TGF- $\beta$ in these cells. Next, we tested whether PKC activation in these cells is reliant on $\mathrm{mTORC1}$ signaling. We found that inhibition of PKC did not decrease the TGF- $\beta$-mediated phosphorylation of S6K1; however, TGF- $\beta$-mediated activation of ribosomal protein 6 (S6) was abolished by PKC inhibition (Figure 3 A). Similar results were obtained when the other pan-PKC inhibitor Bis IX was used (Figure $3 \mathrm{~B}$ ). These results are consistent with the previous finding that PKC can phosphorylate S6 independently of mTORC1 activity [20, 21].

We finally determined whether inhibition of PKC activity can attenuate in vivo metastasis of DAN cells using a xenograft assay. Firefly luciferase labeled DAN cells were injected into the tail vein of $p 53^{-1}$ mice. The mice were injected with either DMSO or G06983 via the tail vein every day for 28 days and the cells were traced every week for 4 weeks via bioluminescence imaging. Whereas DAN cells formed robust tumors in the hind limbs (Figure 4 A), treatment with Go6983 significantly attenuated these tumors (Figure 4 B). After euthanasia bones were imaged for metastatic lesions, and Go69983 treatment significantly inhib- ited metastatic lesions in the bones (Figure $4 \mathrm{C}$ ). Taken together, our results indicated that inhibition of PKC activity can inhibit tumorigenesis and metastatic progression of bone cancer.

\section{Discussion}

Protein kinase $C$ is a prototypical class of serine/threonine kinases that consists of 10 members, which are products of 9 different genes [4] Extensive work established that PKC is involved in multiple signaling pathways that regulate cell function, including growth, proliferation, differentiation and survival, and dysregulation of PKC activity is often associated with a number of diseases, including cancer and metabolic disorders [4]. Earlier studies have shown PKC regulates eukaryotic translational machinery by activating cellular translation [22-24].

The PKC inhibitors have been sparsely tested in clinical trials partly because of the lack of scientific validation of PKC isoforms being valid targets in cancer given that most studies are correlative at best. Midostaurin was the first PKC inhibitor tested in clinical trials against cancer [25]; however, the results have not been favorable [26]. Enzastaurin had a favorable outcome in phase II trials [27], but was halted mid-way through a phase III trial because of failure to meet the primary end point [28]. So there remains a valid reason for both validating PKC isoforms and their activation as direct targets in different cancers, as well
A
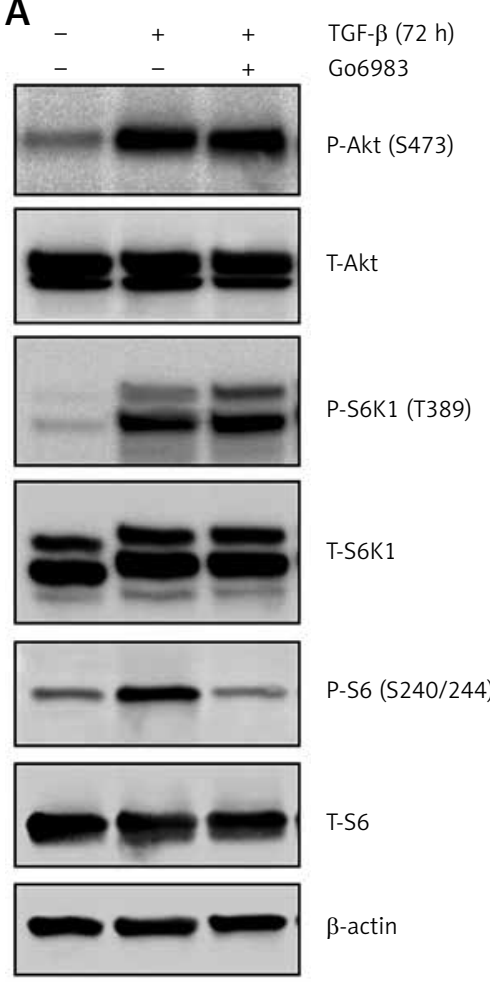

B

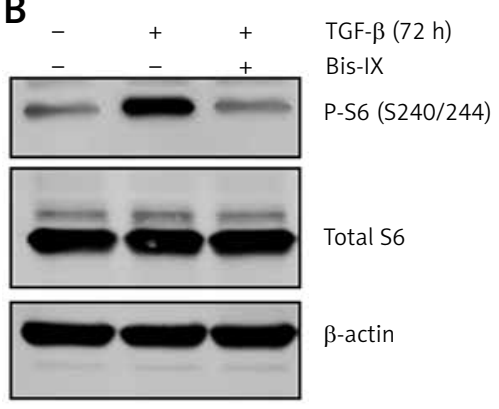

Figure 3. TGF- $\beta$-treatment-mediated PKC activation results in increased phosphorylation of ribosomal protein S6. Immunoblot analysis of P-S6K1 and P-S6 in cell lysates obtained from DAN cells treated with TGF- $\beta$ for $72 \mathrm{~h} \pm$ PKC inhibitors Go6983 (A) or Bis IX (B). The blots were stripped and re-probed with anti- $\beta$-actin in each case to confirm equal loading. Representative blots from three independent experiments are shown 


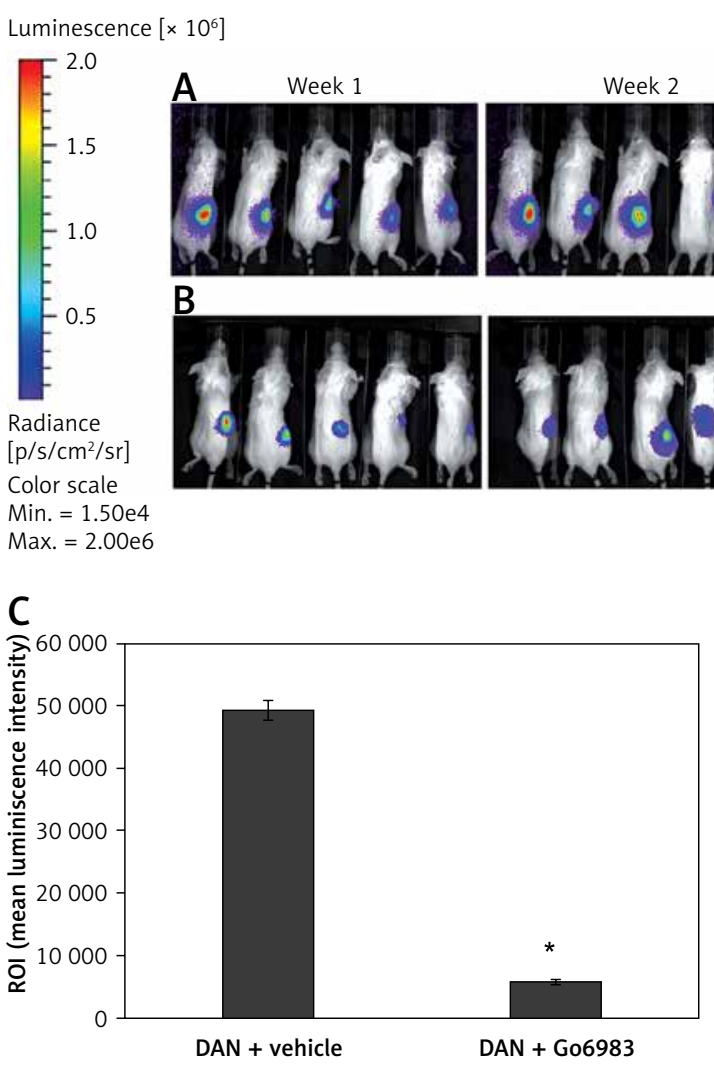

as in finding more potent inhibitors. The role of PKC as a tumor promoter or a suppressor is still not established. While several studies have shown that activation of PKC confers tumor formation, metastasis and invasion, some studies reported that inactive mutations in $P K C$ are associated with disease progression in multiple cancer types [24, 29, 30]. Together, these studies show enormous complexities in understanding the role of PKC in cancer and suggest that the function of PKC as a tumor promoter or a tumor suppressor highly depends on the context of specific oncogenic alterations.

In conclusion, in the current study we saw that PKC activation results in global upregulation of translation in osteosarcoma cells and that potentiates metastasis in vivo. Importantly, treatment with the PKC inhibitor G06983 results in suppression of P-S6, attenuates global translational upregulation, and reduces the potential of these cells to metastasize in vivo. Even though these are critical findings, the translational potential can actually be realized only when it is determined which isoform of PKC is being affected by Go6983. Moreover, it will be imperative to determine toxicity as well as changes in overall gene expression by RNA sequencing.

\section{Conflict of interest}

The authors declare no conflict of interest.
Figure 4. PKC activation correlates with metastatic potential of DAN cells in vivo. A, B - Firefly luciferase expressing DAN cells were intravenously injected subcutaneously via the tail vein in $p 53^{-/-}$mice. From the second day, mice were injected with either DMSO (A) or $1 \mathrm{nM}$ of G06983 (B). Mice were imaged by in vivo luciferase imaging up to 4 weeks to detect tumor formation. C - After euthanasia, bones from DMSO (vehicle) treated and Go6983 treated mice were excised and imaged by bioluminescence imaging to detect metastatic lesions. The mean luminescence intensity of the region of interest (ROI) is shown

${ }^{*} P<0.05$.

\section{References}

1. Patel LR, Camacho DF, Shiozawa Y, et al. Mechanisms of cancer cell metastasis to the bone: a multistep process. Future Oncol 2011; 7: 1285-97.

2. Gutierrez-Uzquiza A, Lopez-Haber C, Jernigan DL, et al. PKCepsilon is an essential mediator of prostate cancer bone metastasis. Mol Cancer Res Mcr 2015; 13: 1336-46.

3. Pan HL, Zhang YQ, Zhao ZQ. Involvement of lysophosphatidic acid in bone cancer pain by potentiation of TRPV1 via PKC $\varepsilon$ pathway in dorsal root ganglion neurons. Mol Pain 2010; 6: 85.

4. Griner EM, Kazanietz MG. Protein kinase $C$ and other diacylglycerol effectors in cancer. Nat Rev Cancer 2007; 7: 281-94.

5. Reyland ME. Protein kinase C isoforms: multi-functional regulators of cell life and death. Front Biosci 2009; 14: 2386-99.

6. Gavrielides MV, Frijhoff AF, Conti CJ, Kazanietz MG. Protein kinase $C$ and prostate carcinogenesis: targeting the cell cycle and apoptotic mechanisms. Curr Drug Targets 2004; 5: 431-43.

7. Jain $\mathrm{K}$, Basu $\mathrm{A}$. The multifunctional protein kinase $\mathrm{C}-\varepsilon$ in cancer development and progression. Cancers 2014; 6: 860-78.

8. Gorin MA, Pan Q. Protein kinase $C \varepsilon$ : an oncogene and emerging tumor biomarker. Mol Cancer 2009; 8: 9.

9. Pan Q, Bao LW, Kleer CG, et al. Protein kinase C epsilon is a predictive biomarker of aggressive breast cancer and a validated target for RNA interference anticancer therapy. Cancer Res 2005; 65: 8366-71.

10. Pan Q, Li WB, Teknos TN, Merajver SD. Targeted disruption of protein kinase $C \varepsilon$ reduces cell invasion and motility through inactivation of RhoA and RhoC GTPases in head and neck squamous cell carcinoma. Cancer Res 2006; 66: 9379-84. 
11. Bae KM, Wang $\mathrm{H}$, Jiang $\mathrm{G}$, et al. Protein kinase $\mathrm{C}$ epsilon is overexpressed in primary human non-small cell lung cancers and functionally required for proliferation of non-small cell lung cancer cells in a p21/Cip1-dependent manner. Cancer Res 2007; 67: 6053-63.

12. Aziz MH, Manoharan HT, Church DR, et al. Protein kinase $C \varepsilon$ interacts with signal transducers and activators of transcription 3 (Stat3), phosphorylates Stat3Ser727, and regulates its constitutive activation in prostate cancer. Cancer Res 2007; 67: 8828-38.

13. Wang HB, Gutierrezuzquiza A, Garg R, et al. Transcriptional regulation of oncogenic protein kinase $\mathrm{C} \varepsilon$ (PKC $\varepsilon$ ) by STAT1 and Sp1 proteins. J Biol Chem 2014; 289: 19823-38.

14. Dann SG, Golas J, Miranda M, et al. p120 catenin is a key effector of a Ras-PKC $\varepsilon$ oncogenic signaling axis. Oncogene 2014; 33: 1385-94.

15. Caino MC, Lopezhaber C, Kim J, et al. Proteins kinase $C|[e p s i v]|$ is required for non-small cell lung carcinoma growth and regulates the expression of apoptotic genes. Oncogene 2012; 31: 2593-600.

16. Jain K, Basu A. Protein kinase $C-\varepsilon$ promotes EMT in breast cancer. Breast Cancer 2014; 8: 61-7.

17. D'Oronzo S, Brown J, Coleman R. The role of biomarkers in the management of bone-homing malignancies. J Bone Oncol 2017; 9: 1-9.

18. Fedorov YV, Jones NC, Olwin BB. Atypical protein kinase Cs are the Ras effectors that mediate repression of myogenic satellite cell differentiation. Mol Cell Biol 2002; 22: 1140-9.

19. Xing F, Okuda H, Watabe M, et al. Hypoxia-induced Jagged2 promotes breast cancer metastasis and self-renewal of cancer stem-like cells. Oncogene 2011; 30: 4075-86.

20. Valovka T, Verdier FR, Zhyvoloup A, et al. Protein kinase C phosphorylates ribosomal protein S6 kinase betall and regulates its subcellular localization. Mol Cell Biol 2003; 23: 852-63.

21. Martin PM, Aeder SE, Chrestensen CA, et al. Phorbol 12-myristate 13 -acetate and serum synergize to promote rapamycin-insensitive cell proliferation via protein kinase C-eta. Oncogene 2007; 26: 407-14.

22. Tejedamuñoz N, Gonzálezaguilar H, Santoyoramos P, et al. Glycogen synthase kinase 3 beta is positively regulated by protein kinase $C \zeta$-mediated phosphorylation induced by Wnt agonists. Mol Cell Biol 2015; 36: 731-41.

23. Chen RH, Ding WV, Mccormick F. Wnt signaling to beta-catenin involves two interactive components. Glycogen synthase kinase-3beta inhibition and activation of protein kinase C. J Biol Chem 2000; 275: 17894-9.

24. Mochlyrosen D, Das K, Grimes KV. Protein kinase C, an elusive therapeutic target? Nat Rev Drug Discov 2012; 11: 937-57.

25. Meyer T, Regenass U, Fabbro D, et al. A derivative of staurosporine (CGP 41251) shows selectivity for protein kinase $C$ inhibition and in vitro anti-proliferative as well as in vivo anti-tumor activity. Int J Cancer 1989; 43: 851-6.

26. Millward MJ, House C, Bowtell D, et al. The multikinase inhibitor midostaurin (PKC412A) lacks activity in metastatic melanoma: a phase IIA clinical and biologic study. Br J Cancer 2006; 95: 829-34.

27. Jirousek MR, Gillig JR, Gonzalez CM, et al. (S)-13-[(dimethylamino)methyl]-10,11,14,15-tetrahydro-4,9:16, 21-dimetheno- $1 \mathrm{H}, 13 \mathrm{H}$-dibenzo[e,k]pyrrolo[3,4-h][1,4,13]oxadiazacyclohexadecene-1,3(2H)-d ione(LY333531) and related analogues: isozyme selective inhibitors of protein kinase C beta. J Med Chem 1996; 39: 2664-71.
28. Chen YB, LaCasce AS. Enzastaurin. Expert Opin Investig Drugs 2008; 17: 939-44.

29. Garg R, Benedetti LG, Abera MB, et al. Protein kinase $C$ and cancer: what we know and what we do not. Oncogene 2014; 33: 5225-37.

30. Antal CE, Hudson AM, Kang E, et al. Cancer-associated protein kinase $C$ mutations reveal kinase's role as tumor suppressor. Cell 2015; 160: 489-502. 